\title{
LESÕES DO TRATO GASTROINTESTINAL NA SÍNDROME DA IMUNODEFICIÊNCIA ADQUIRIDA: ESTUDO DE 45 NECRÓPSIAS CONSECUTIVAS
}

\author{
Maria Goretti Freire de Carvalho, Maria Aparecida Marchesan Rodrigues, \\ Mariângela Ester Marques, Marcellọ Franco e Mário Rubens Montenegro
}

\begin{abstract}
Com objetivo de estudar a frequência e etiologia das lesões do tubo digestivo na Sindrome da Imunodeficiência Adquirida (AIDS), foram analisadas retrospectivamente 45 necrópsias consecutivas de pacientes adultos portadores do vírus da AIDS. Lesões macroscópicas e cortes histolögicos de amostras da boca à região anal foram estudados, sendo as lâminas coradas por HE, métodos histoquímicos ou imunohistoquímicos. Trinta esete $(82,3 \%)$ pacientes apresentaram lesões no tubo digestivo. O local mais freqüente de lesões foi a boca $(73,3 \%)$, seguido do cólon (55,5\%). Lesões múltiplas foram identificadas em 17 (37,7\%) casos. O diagnóstico mais prevalente foi infeç̧ẫo pelo citomegalovírus $(35,7 \%)$ identificado predominantemente no cólon. Candidíase foi mais freqüente na boca $(26,6 \%)$ e infecção herpética no esôfago $(8,8 \%)$. Verificou-se leucoplasia pilosa oral em 16 (35,5\%) e neoplasias em sete (15,5\%). As neoplasias incluiram quatro sarcomas de Kaposi, dois carcinomas intramucosos anais e um linfoma gástrico. Os dados do presente estudo confirmam a importância do trato gastrointestinal como sede de alterações patológicas relacionadas à AIDS.
\end{abstract}

Palavras-chaves: Síndrome da imunodeficiência adquirida. Infeç̧ões oportunistas. Trato gastrointestinal. Necrópsia.

O comprometimento do trato gastrointestinal (TGI) constitui uma das mais comuns e debilitantes conseqüências da AIDS e afeta de 50 a $100 \%$ dos casos $^{16}$. De fato, a candidíase oro-esofágica é um dos primeiros sinais da doença e a diarréia uma de suas mais freqüentes manifestações, ocorrendo entre 50 e $90 \%$ dos pacientes ${ }^{8} 1624$.

Entre os agentes responsáveis pela agressão ao TGI, além dos oportunistas como a Cândida, outros fungos, vírus, bactérias e protozoários, está o próprio HIV ${ }^{15}$. O sarcoma de Kaposi, linfomas e carcinomas também costumam colaborar paraagravar os sinais e sintomas gastrointestinais da AIDS.

A etiologia das complicações da AIDS, no entanto, varia em diferentes regiões, sendo raras as publicações que focalizem a freqüência e tipo das álterações do TGI em necrópsias brasileiras ${ }^{4} 12$.

Neste estudo, apresentamos e discutimos o que

\footnotetext{
Departamento de Patologia, Faculdade de Medicina da Universidade Estadual Paulista (UNESP) Botucatu, SP.

Endereço para correspondência: Dra. Maria Aparecida M. Rodrigues. Depto. de Patologia/FM/UNESP, Distrito de Rubião Junior s/n 18618-000 Botucatu, SP.

Recebido para publicação em $06 / 01 / 94$.
}

foi observado no TGI, em necrópsias de pacientes portadores do vírus da AIDS que faleceram no Hospital das Clínicas da Faculdade de Medicina de Botucatu - UNESP.

\section{MATERIAL E MÉTODOS}

De outubro de 1988 a junho de 1992, foram realizadas no Departamento de Patologia da Faculdade de Medicina de Botucatu, 45 necrópsias de pacientes portadores do vírus da AIDS, que constituem o objeto deste estudo. Revisamos os prontuários e relatórios macroscópicos, sendo que o estudo histológico foi feito em lâminas arquivadas. Dispúnhamos de lâminas de todos os segmentos do TGI, desde a boca até a região anal, em todos os casos. As lâminas foram coradas por HE e cortes selecionados foram corados por Fite-Faraco, prata metenamina de Gomori-Grocott, Giemsa, ZiehlNielsen e PAS. Quando necessário, utilizou-se a técnica da avidina-biotina-peroxidase e anticorpos monoclonais anti-HPV, antiCD20 e antiCD45RO (DAKO, LTD, High, Wycombe, UK). 
Carvalho MGF, Rodrigues MAM, Marques ME, Franco M, Montenegro MR. Lesões do trato gastrointestinal na Sindrome da Imunodeficiência Aquirida: estudo de 45 necrópsias consecutivas. Revista da Sociedade Brasileira de Medicina Tropical 27:135-141, jul-set, 1994.

\section{RESULTADOS}

O diagnóstico de AIDS foi feito de acordo com os critérios previamente definidos para esta entidade ${ }^{6}$ 11. O perfil imunológico avaliado em 15 pacientes demonstrou em 13 relação $\mathrm{CD} 4 / \mathrm{CD} 8$ menor que 0,70 .

Todos os pacientes com exceção de um eram adultos; destes, 32 eram do sexo masculino, sendo que a idade variou entre 23 e 58 anos. Os grupos de risco foram: homossexuais (11), viciados em drogas (8), bissexuais (4) e indeterminado (9). As 12 mulheres tinham entre 26 e 68 anos de idade e pertenciam aos seguintes grupos de risco: prostituição e drogas (4), prostituição (1), viciadas em drogas (4), parceiras de aidéticos (3). A única criança estudada era uma menina de 10 anos, que recebeu plasma por desidratação há 7 anos.

Quanto às alterações clínicas, a diarréia foi a mais prevalente, diagnosticada em 14 pacientes. Ocasionalmente foram observadas náuseas e vômitos. Um paciente apresentou sangramento gastrointestinal maciço e outro melena.

Nas 45 necrópsias estudadas havia lesões macro elou microscópicas do tubo digestivo em $82,3 \%$. Quanto aos demais orgãos verificou-se comprometimento predominante dos pulmões em
19 casos, sendo 6 por infecção por Pneumocystis carinii e 5 pelo bacilo de Koch.

O sistema nervoso central estava acometido em 14 casos, sendo 11 por toxoplasmose. $O$ óbito foi relacionado a lesões do tubo digestivo em 4 casos, todos com lesões intestinais extensas necrosantes $e$ hemorrágicas, 3 por citomegalovírus (CMV) e 1 com gastroenterocolite hemorrágica aguda de etiologia não esclarecida, que faleceu com quadro de hemorragia gastrointestinal maciça.

As Tabelas 1 e 2 referem-se à freqüência e etiologia das lesões infecciosas e neoplásicas do tubo digestivo por sede anatômica.

A boca foi o segmento mais freqüentemente acometido, sendo a lesão mais freqüente a leucoplasia pilosa observada em 16 casos (Figura 1). Em um de cinco casos pesquisados, foi identificado papilomavírus (HPV) por imunoperoxidade (Figura 2). A candidíase foi diagnosticada em 12 casos, seguida de sarcoma de Kaposi em três casos.

O cólon foi comprometido em 25 casos, sendo a etiologia definida em 23. Havia em 15 casos infecção pelo CMV. Nestes a mucosa apresentava, ao exame macroscópico, alterações focais caracterizadas por pequenas erosões ou úlceras superficiais com halo hemorrágico. Em três casos as lesões eram extensas, necro-hemorrágicas.

Tabela 1 - Frequiência e etiologia de lesões infecciosas e neoplásicas do tubo digestivo proximal em 45 pacientes com AIDS.

\begin{tabular}{|c|c|c|c|c|c|c|}
\hline \multirow{3}{*}{ Lesão } & \multicolumn{6}{|c|}{ Local } \\
\hline & \multicolumn{2}{|c|}{ boca } & \multicolumn{2}{|c|}{ esôfago } & \multicolumn{2}{|c|}{ estômago } \\
\hline & $n$ & $\%$ & $\mathrm{n}$ & $\%$ & $\mathbf{n}$ & $\%$ \\
\hline \multicolumn{7}{|l|}{ Infecciosa/agente } \\
\hline$H P V-E B V$ & 16 & 35,5 & - & - & $\rightarrow$ & - \\
\hline$C M V$ & - & $\div$ & 4 & 8,8 & 8 & 17,7 \\
\hline Candida albicans & 12 & 26,6 & 3 & 6,6 & - & - \\
\hline Helicobacter pylori & - & - & - & - & 2 & 4,4 \\
\hline Criptococcus & - & - & - & - & 1 & 2,2 \\
\hline Histoplasma & 2 & 4,4 & - & - & - & - \\
\hline Herpes simplex & - & - & 4 & 8,8 & - & - \\
\hline Não definida & - & - & 2 & 4,4 & - & - \\
\hline \multicolumn{7}{|l|}{ Neoplasia } \\
\hline linfoma & - & - & - & - & 1 & 2,2 \\
\hline sarcoma de Kaposi & 3 & 6,6 & - & - & 1 & 2,2 \\
\hline Total & 33 & 73,3 & 13 & 28,8 & 13 & 28,8 \\
\hline
\end{tabular}

$H P V-E B V=$ Papilomavírus-vírus de Epstein-Barr

$C M V=$ Citomegalovirus 
Carvalho MGF, Rodrigues MAM, Marques ME, Franco M, Montenegro MR. Lesões do trato gastrointestinal na Síndrome da Imunodeficiência Aquirida: estudo de 45 necrópsias consecutivas. Revista da Sociedade Brasileira de Medicina Tropical 27:135-141, jul-set, 1994.

Tabela 2 - Freqüência e etiologia de lesões infecciosas e neoplásicas do tubo digestivo distal em 45 pacientes com AIDS.

\begin{tabular}{|c|c|c|c|c|c|c|c|c|}
\hline \multirow{3}{*}{ Lesão } & \multicolumn{8}{|c|}{ Local } \\
\hline & \multicolumn{2}{|c|}{ i. delgado } & \multicolumn{2}{|c|}{ apêndice } & \multicolumn{2}{|c|}{ cólon } & \multicolumn{2}{|c|}{ reto/ânus } \\
\hline & $\mathrm{n}$ & $\%$ & $\mathrm{n}$ & $\%$ & $\mathrm{n}$ & $\%$ & $\mathrm{n}$ & $\%$ \\
\hline \multicolumn{9}{|l|}{ Infecciosa/agente } \\
\hline$C M V$ & 11 & 24,4 & - & - & 15 & 33,3 & 1 & 2,2 \\
\hline Candida albicans & - & - & - & - & 1 & 2,2 & - & - \\
\hline$M A I$ & 2 & 4,4 & - & - & 2 & 4,4 & - & - \\
\hline Criptosporidium & 2 & 4,4 & 1 & 2,2 & 1 & 2,2 & - & - \\
\hline Criptococcus & 2 & 4,4 & 1 & 2,2 & 2 & 4,4 & - & - \\
\hline Histoplasma & - & - & - & - & 1 & 2,2 & - & - \\
\hline M. tuberculosis & - & - & - & - & 1 & 2,2 & - & - \\
\hline S. stercolaris & 1 & 2,2 & - & - & - & - & - & - \\
\hline Papilomavirus & - & - & - & - & - & - & 2 & 4,4 \\
\hline Helicobacter & - & - & 1 & 2,2 & - & - & - & - \\
\hline Não definida & - & - & - & - & 2 & 4,4 & - & - \\
\hline \multicolumn{9}{|l|}{ Neoplasia } \\
\hline $\mathrm{Ca}$ intramucoso & - & - & - & - & - & - & 2 & 4,4 \\
\hline sarcoma de Kaposi & 3 & 6,6 & - & - & - & - & - & - \\
\hline Total & 21 & 46,6 & 3 & 6,6 & 25 & 55,5 & 5 & 11,1 \\
\hline
\end{tabular}

$C M V=$ Citomegalovírus

$M A I=M$, avium intracellulare

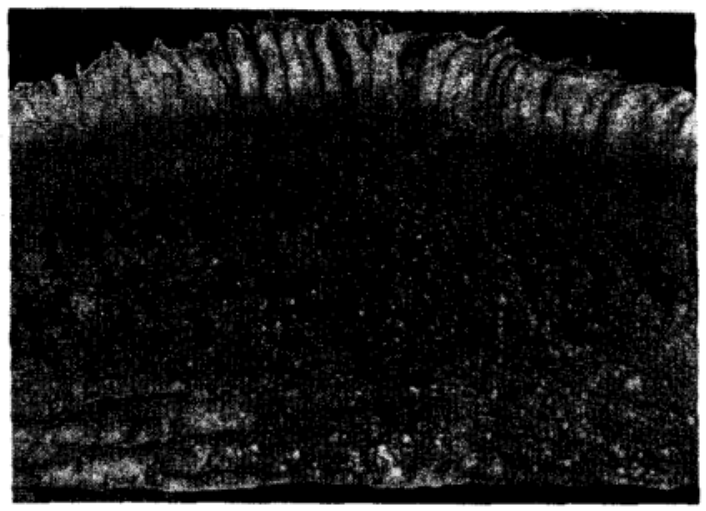

Figura 1 - Leucoplasia pilosa oral. Corte longitudinal de língua exibindo mucosa espessa, de aspecto piloso.

Histologicamente, foram identificadas partículas virais predominantemente no endotélio de vasos da lâmina própria ou da submucosa e, ocasionalmente, no epitélio ou na mucosa adjacente às lesões (Figura 3).

Chamou atenção a elevada freqüência de lesões causadas pelo citomegalovírus, pela Cândida e pelo complexo Papilomavírus-vírus de Epstein-Barr (HPV-EBV), conforme demonstram as Tabelas $1 \mathrm{e}$

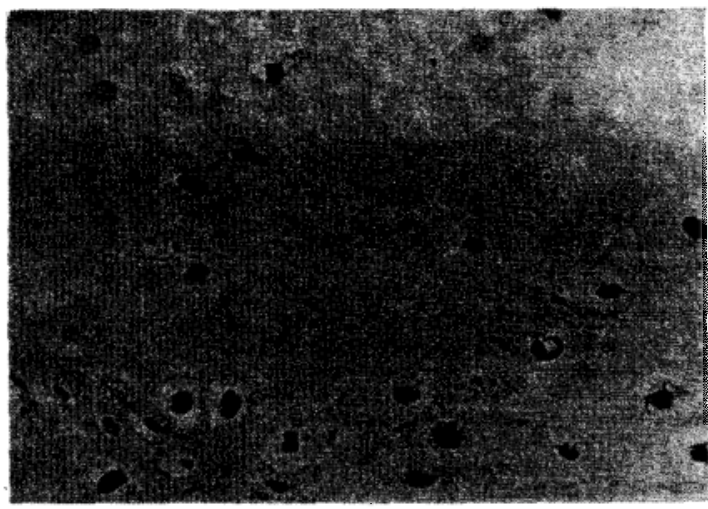

Figura 2 - Leucoplasia pilosa oral. Imuno-reatividade paraHPVem núcleos de células pavimentosas (ABC+anti-HPVx 400).

2. Em apenas quatro casos houve lesões por Herpes simplex (todas no esôfago) e em duas por papilomavírus (na mucosa anal). Observamos lesões criptocócicas no estômago (Figura 4) e histoplasmóticas (boca e cólon). O Mycobacterium avium intracellulare (MAI) foi encontrado em dois casos, com lesões no intestino delgado e cólon, e o Bacilo de Koch em um caso, no cólon. Não foi feita cultura para distinguir o MAI do bacilo de Koch. $\mathrm{O}$ 
Carvalho MGF, Rodrigues MAM, Marques ME, Franco M, Montenegro MR. Lesões do trato gastrointestinal na Sindrome da Imunodeficiência Aquirida: estudo de 45 necrópsias consecutivas. Revista da Sociedade Brasileira de Medicina Tropical 27:135-141, jul-set, 1994.

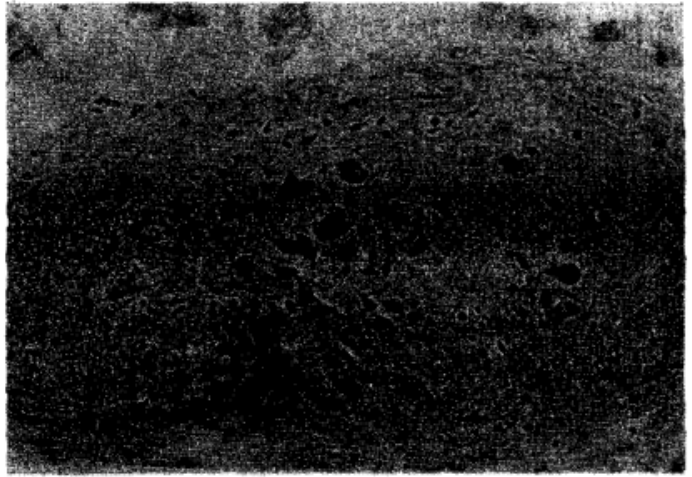

Figura 3 - Células endoteliais com inclusões de CMV em vaso de submucosa intestinal (HE $x$ 2000).

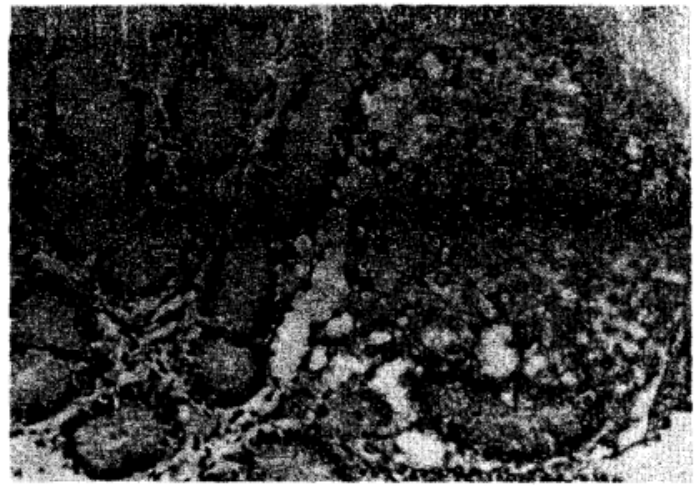

Figura 4 - Criptococcus neoformans no córion da mucosa gástrica com ausência de reação inflamatória (Mucicarmin x 200).

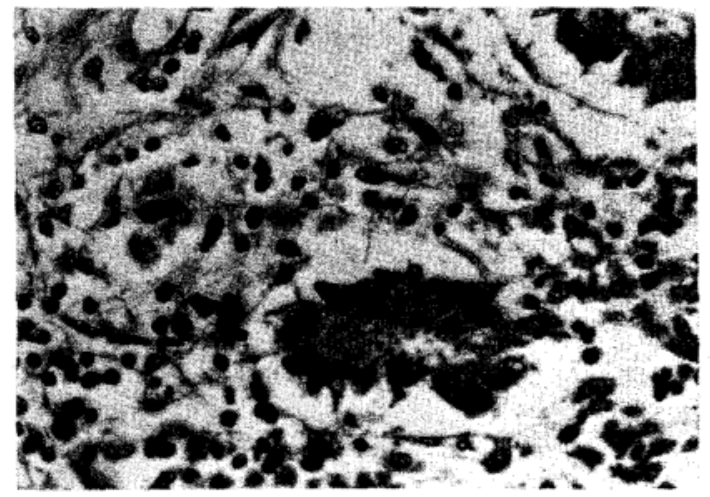

Figura 5 - Criptosporidium em cripta de glândula intestinal (HE $x$ 400).

diagnóstico diferencial foi baseado em critérios morfológicos. As lesões herpéticas eram em geral úlceras rasas, focais, irregulares; em um caso havia extensas lesões pseudomembranosas. Nos casos de $\mathrm{MAI}$, as lesões foram identificadas somente ao exame histológico e corresponderam à disseminação hematogênica e/ou linfática do agente para os intestinos.

No caso de tuberculose, o órgão mais comprometido foi o pulmão, com disseminação sistêmica e lesões miliares em serosa e mucosa dos intestinos delgado e grosso. Em apenas dois casos identificamos Helicobacter pylori na mucosa gástrica, que era de aspecto atrófico.

Houve três casos de criptosporidiose; neles não foram identificadas lesőes macroscópicas, sendo o diagnóstico feito pela identificação histológica do agente (Figura 5). Em um caso havì infecção por Strongiloides stercoralis; as larvas foram observadas nos cortes histológicos do jejuno.

Foram ainda observadas outras lesões no estômago (sarcoma de Kaposi e linfoma), no intestino delgado (sarcoma de Kaposi) e na região anal (dois casos de carcinoma espinocelular intramucoso). Muitos dos pacientes apresentaram lesões de mais de uma etiologia. As associações mais freqüentes foram citomegalovirus com candidíase ( 5 casos), com leucoplasia pilosa ( 3 casos) e com sarcoma de Kaposi (3 casos). Entretanto, em oito pacientes não foram identificados agentes etiológicos no trato gastrointestinal. As alterações encontradas, nestes pacientes, corresponderam a infiltrado inflamatório com predomínio de plasmócitos no cório da mucosa intestinal, havia atrofia do tecido linfóide intestinal e apendicular, discreta atrofia de vilosidades intestinais e hemossiderose localizada. Dois pacientes exibiram ulcerações esofágicas e colônicas, sem que o agente etiológico tivesse sido identificado.

\section{DISCUSSÃ̃O}

Pacientes com AIDS apresentam alterações da imunidade celular, dependentes da destruição de células $\mathrm{T}$ auxiliares, por mecanismos direta ou indiretamente causados pelo HIV, bem como por alterações de células apresentadoras de antígenos ${ }^{18}$. Mais ainda, apresentam deficiências nutricionais, como de selênio, que podem exercer impacto negativo sobre o já comprometido sistema imune ${ }^{8}$, aumentando a susceptibilidade a infecções oportunistas e neoplasias que, em conjunto, correspondem às principais causas de morte destes pacientes $^{19}$. 
Carvalho MGF, Rodrigues MAM, Marques ME, Franco M, Montenegro MR. Lesões do trato gastrointestinal na Sindrome da Imunodeficiência Aquirida: estudo de 45 necrópsias consecutivas. Revista da Sociedade Brasileira de Medicina Tropical 27:135-141, jul-set, 1994.

As infecções oportunistas do TGI comprometem $\mathrm{da}$ boca ao ânus e correspondem àquelas produzidas por protozoários (Cryptosporidium, Microsporidium, Isospora belli, Giardia), vírus (CMV, Herpes simplex), fungos (Candida sp. Histoplasma capsulatum, Cryptococcus neoformans) e menos freqüentemente por bactérias tais como complexo MAI, M. tuberculosis, $H$. pylori, Campilobacter sp, Salmonella sp 1220242526 .

Outras infecções oportunistas que comprometem pacientes portadores do vírus HIV são produzidas pelo $P$. carinii e pelo Toxoplasma gondii que, embora muito freqüentes em outros órgãos, só raramente comprometem o tubo digestivo ${ }^{17}$. Em 45 pacientes estudados, verificamos 12 casos de toxoplasmose (11 do SNC), sem que, no entanto, observássemos acometimento do TGI.

Dos 37 casos com alterações do TGI, 91\% foram atribuídos a infecções.

$O$. segmento do tubo gastrointestinal mais acometido foi a boca $(73,3 \%)$, sendo a lesão mais freqüente a leucoplasia pilosa, uma lesão específica descrita nesses pacientes, como placas brancacentas com finas projeções digitiformes, localizadas nas faces laterais da língua. $\mathrm{O}$ padrão histológico da lesão corresponde ao de um condiloma plano, cujo epitélio mostra acantose, paraceratose e projeções de ceratina para a superfície; atipias coilocíticas são proeminentes, bem como a presença de células baloniformes. Sua etiologia parece estar relacionada ao HPV e ao vírus EBV ${ }^{1923}$. Emum de cinco casos pesquisados por imunohistoquímica pudemos demonstrar a presença do HPV.

Após a boca, o cólon $(55,5 \%)$ e o intestino delgado $(46,6 \%)$ foram os segmentos mais afetados, neles havendo lesões decorrentes predominantemente da infecção pelo CMV. Este virus parece ser o mais comum agente infeccioso oportunístico nos pacientes com AIDS ${ }^{16}{ }^{19}$. Produz lesões inflamatórias ulcerativas ou pseudomembranosas que predominam no cólon e podem evoluir com complicações tais como a perfuração ${ }^{2} 81928$. Dos nossos 21 casos de citomegalovirose, 10 pacientes eram homossexuais.

Embora o Cryptosporidium seja considerado agente infeccioso de freqüência semelhante ao $\mathrm{CMV}^{1216}$, observamos este protozoário em apenas quatro pacientes, fato talvezjustificado por estarmos analisando material de necrópsia. Este protozoário parasita as células epiteliais superficiais ou do fundo das criptas da mucosa intestinal. Tem preferência pelo intestino delgado, causando nesses pacientes diarréia grave com distúrbios hidroeletrolíticos ${ }^{28}$. É parasita de difícil visualização, podendo passar despercebido, principalmente ao exame microscópico de material de autópsia que freqüentemente apresenta alterações autolíticas e de descamação. A coloração pelo Giemsa facilita sua identificação.

Outra infecções menos comuns comprometeram o tubo digestivo baixo, tais como criptococose, micobacteriose e estrongiloidíase; todas ocorreram como infecções oportunistas, associadas ao CMV, indicando o avançado grau de deficiência imunológica desses pacientes.

Infecções acometendo o trato digestivo alto corresponderam principalmente àquelas produzidas pela Candida sp e pelo Herpes simplex, que são causas comuns de disfagia e odinofagia, freqüentes nestes pacientes. A candidíase afeta predominantemente as mucosas bucal e esofágica ${ }^{5}$ 13 aparece sob a forma de placas brancacentas ou de pseudomembranas, raramente invadindo e produzindo lesões sistêmicas ${ }^{16}$. Já as infecções herpéticas caracterizam-se por ulcerações profundas, freqüentes no terço inferior do esôfago, exibindo ao exame histológico, células epiteliais isoladas ou sinciciais, contendo inclusões eosinofílicas, com aspecto de vidro fosco ${ }^{528}$.

Das lesões neoplásicas observadas, chamou atenção a associação do HPV com os carcinomas anais intramucosos, evidenciando mais uma vez a estreita relação existente entre estas patologias, 0 HIV e hábitos homossexuais ${ }^{1926}$. Do mesmo modo, - sarcoma de Kaposi afeta principalmente homossexuais e guarda estreita relação com a infecção pelo $\mathrm{CMV}$, acreditando alguns que o vírus interfere em sua gênese ${ }^{132}$. Esta neoplasia ocorre em 30\% dos pacientes aidéticos, acometendo principalmente a pele, com disseminação para o tubo digestivo em 40-50\% dos casos ${ }^{23}$. Correspondem a máculas ou pápulas avermelhadas, por vezes umbilicadas, histologicamente semelhantes ao sarcoma de Kaposi clássico. Dos 45 pacientes que estudamos, seis apresentaram sarcoma de Kaposi cutâneo com disseminação para o tubo digestivo em quatro. Esses pacientes exibiam também infecção pelo CMV e três eram homossexuais. 
Carvalho MGF, Rodrigues MAM, Marques ME, Franco M, Montenegro MR. Lesóes do trato gastrointestinal na Síndrome da Imunodeficiência Aquirida: estudo de 45 necrópsias consecutivas. Revista da Sociedade Brasileira de Medicina Tropical 27:135-141, jul-set, 1994.

Lesões inflamatórias inespecíficas, caracterizadas por afluxo linfo-plasmocitário ao lado de discreta atrofia vilositária, ou ulcerações rasas da mucosa, descritas anteriormente em humanos ou em pesquisa experimental ${ }^{3} 5915202425$ foram observadas em $17,7 \%$ de nossos pacientes. A dificuldade em correlacionar sintomas ou achados morfológicos com a presença de patógenos conhecidos sugere a existência de uma gastroenteropatia associada ao $\mathrm{HIV}^{7}{ }^{10}$. Nelson e coll $^{22}$ detectaram por hibridização in situ, células epiteliais das criptas intestinais infectadas por HIV. Numerosas pesquisas vêm demonstrando que outras cepas deste vírus são capazes de infectar células epiteliais da superfície colônica, células enterocromafins e células mononucleares do córion da mucosa ${ }^{14} 27$. No entanto, a pesquisa de agentes oportunistas deve ser exaustivamente realizada, tanto em material de patologia cinirgica como de necrópsia, tendo em vista que estes agentes agravam o quadro intestinal e são passíveis de controle, com tratamento adequado. Deve ser lembrado que alguns destes agentes como o Crytosporidium e o Microsporidium são de difícil diagnóstico, sendo necessário pesquisa dirigida, para identificá- $\operatorname{los}^{21}$. Além disso, pacientes aidéticos mostram diminuição da relação de linfócitos $T$ (CD4/CD8), tanto sistêmica quanto local, explicando a prevalência de infecçōes oportunistas e de neoplasias no TGT21. Estudamos esta relação no sangue periférico de nossos pacientes, que se mostrou alterada em todos aqueles que apresentaram lesões oportunistas do tubo digestivo. Estudos recentes propõem uma inter-ręlação entre o sistema imune e a estrutura da mucosa intestinal, tendo-se demonstrado que os enterócitos possuem receptores para células CD4, e que estas parecem controlar sua proliferação e maturação ${ }^{714}$.

\section{SUMMARY}

This study was designed to evaluate resiropectively the frequency and etiology of the gastrointestinal (Gl) lesions in 45 consecutive necropsies of adult patients with Acquired Immunodeficiency Syndrome (AIDS). Gross descriptions and histological sections of the GI tract, from mouth to anus, were reviewed. The slides were $H \& E$ stained, and when necessary special stains and immunohistochemicalmethods were also employed. There were lesions in Gl tract in 37 (82.3\%) patients; the mouth was the segment most frequently involved $73.3 \%$ of the cases), followed by the colon (55.5\%). Multiple lesions occurred in 17 (37.7\%) cases. Cytomegalovirus caused colonic lesions in $35.7 \%$ of the cases. Candidiasis was observed in $26.6 \%$ mainly in the mouth and herpes simplex (8.8\%) was the important agent of esophageal lesions. Oral hairy leukoplasia associated with HPV was found in $16(35.5 \%)$ cases. Neoplasia was diagnosed in 7 (15.5\%) cases: four Kaposi's sarcoma, two anal intramucosal carcinomas and one gastric lymphoma. Our data confirm the high frequency and variety of GI tract alterations in AIDS.

Key-words: Acquired immunodeficiency syndrome. Opportunistic infections. Digestive tract. Necropsy.

\section{REFERÊNCIAS BIBLIOGRÁFICAS}

1. Anderson M. Gastroenterological aspects of AIDS in the Third World. Baillière's Clinical Gastroenterology 4:375-383, 1990.

2. Barone JE, Wolkomir AF, Muakkassa FF, Fares II LG. Abdominal pain and anorectal disease in AIDS. Gastroenterology Clinics of North America 17:631638, 1988.

3. Baskerville A, Ramsay A, Cranage MP, Cook N, Cook RW, Dennis MJ, Greenaway PJ, Kitchin PA, Stott EJ. Histopathological changes in simian immunodeficiency virus infection. Journal of Pathology 162:67-75, 1990.

4. Brandão-Mello CE, Basílio-de-Oliveira CA, Fialho F, Bonecker CWC, Gonzaga AL, Pontes EL, Salomão AR. Acometimento gastrointestinal pelo citomegalovírus na síndrome de imunodeficiência adquirida. Arquivos Brasileiros de Medicina 63:9$16,1989$.

5. Cello JP. AIDS and the Gastroenterologist. Scandinavian Journal of Gastroenterology 25(suppl. 175):146-158, 1990.

6. Centers for disease control and prevention. Revised classification system for HIV infection and expanded surveillance case definition for AIDS among adolescents and adults. MMWR Journal American Medical Association 269:729, 1993.

7. Cummins AG, La Brooy JT, Stanley DP, Rowland $\mathbf{R}$, Shearman DJC. Quantitative histological study of enteropathy associated with HIV infection. GUT 31:317-321, 1990.

8. Dworkin B, Wormser GP, Rosenthal WS, Heier SK, Braunstein M, Weiss L, Jankowski R, Levy D, Weiselberg S. Gastrointestinal manifestations of the acquired immunodeficiency syndrome: A review of 22 cases. The American Joumal of Gastroenterology 80:774-778, 1985. 
Carvalho MGF, Rodrigues MAM, Marques ME, Franco M, Montenegro MR. Lesões do trato gastrointestinal na Sindrome da Imunodeficiência Aquirida: estudo de 45 necrópsias consecutivas. Revista da Sociedade Brasileira de Medicina Tropical 27:135-141, jul-set, 1994.

9. Elia C, Oliveira AV, Madi K. Biopsia jejunal em SIDA. Arquivos de Gastroenterologia 26:100-104, 1989.

10. Fantini J, Yahi N, Baghdiguian S, Chermann JC. Human colon epithelial cells productively infected with human immunodeficiency virus show impaired differentiation and altered secretion. Journal of Virology 66:580-585, 1992.

11. Fauci AS, Macher AM, Longo DL, Lane HC, Rook AH, Masur H, Gelmann EP. Acquired immunodeficiency syndrome: epidemiologic, clinical, immunologic, and therapeutic considerations. Annals of Internal Medicine 100:92106, 1984.

12. Fialho F, Basílio-de-Oliveira CA. O aparelho digestivo na síndrome de imunodeficiência adquirida. Considerações anatomopatoiógicas. Anais da Academia Nacional de Medicina 152:136-144, 1992.

13. Gazzard B. AIDS: an overvicw. Baillière's Clinical Gastroenterology 4:259-289, 1990.

14. Heisse C, Dandekar S, Kumar P, Duplantier R, Donovan RM, Kalsted $\mathrm{CH}$. Human immunodeficiency virus infection of enterocytes and monocuclear cells in human jejunal mucosa. Gastroenterology 100:1521-1527, 1991.

15. Heisse C, Vogel P, Miller CJ, Halsted CH, Dandekar S. Simian immunodeficiency virus infection of the gastrointestinal tract of rhesus macaques: functional, pathological and morphological changes. American Journal of Pathology 142:1759-1771, 1983.

16. Janoff EN, Smith PD. Perspectives on gastrointestinal infections in AIDS. Gastroenterology Clinics of North America 17:451-463, 1988.

17. Jautzke G, Sell M, Thalmann U, Janitschke K, Gottschalk J, Shürmann D, Ruf B. Extracerebral toxoplasmosis in AIDS: histological immunohistological findings based on 80 autopsy cases. Pathology Research and Practice 189:428436, 1993.

18. Lim SG, Condez A, Poulter LW. Mucosal macrophage subsets of the gut in HIV: decrease in antigen-presenting cell phenotype. Clinical and Experimental Immunology 92:442-447, 1993.
19. Macho JR. Gastrointestinal surgery in the AIDS patient. Gastroenterology Clinics of North America 17:563-571, 1988.

20. McLoughlin LC, Nord KS, Joshi VV, Oleske JM, Connor EM. Severe gastrointestinal involvement in children with the acquired immunodeficiency syndrome. Journal of Pediatric Gastroenterology and Nutrition 6:517-524, 1987.

21. Michiels JF, Hofman P, Saint Paul MC, Loubière R, Bernard E, Lefichoux Y. Pathological features of intestinal microsporidiosis in HIV positive patients: a report of 13 new cases. Pathology Research and Practive 189:377-383, 1993.

22. Nelson JA, Reynolds-Kohler C, Margaretten W, Wiley CA, Reese CE, Levy JA. Human immunodeficiency virus detected in bowel epithelium from patients with gastrointestinal symptoms. The Lancet 6:259-262, 1988.

23. Pavli $P$, Doe WF. The alimentary tract in disorders of the immune system. $I n$ : Whitehead $\mathrm{R}$ (ed). Gastrointestinal and Oesophageal Pathology. Churchill Livingstone, London p.187-200, 1989.

24. René E, Verdon R, Aids Git Group: Upper gastrointestinal tract infections in AIDS. Baillière's Clinical Gastroenterology 4:339-359, 1990.

25. Rodgers VD, Kagnoff MF. Gastrointestinal manifestations of the acquired immunodeficiency syndrome. The Western Journal of Medicine 146:5767, 1987.

26. Stamm B, Grant JW. Biopsy pathology of the gastrointestinal tract in human immudeficiency virusassociated disease: a 5 year experience in Zurich. Histopathology 13:531-540, 1988.

27. Ullrich R, Zeitz M, Heise W, L'age M, Höffken G, Riecken EO. Small intestinal structure and function in patients infected with human immunodeficiency virus (HIV): evidence for HIV induced enteropathy. Annals of Internal Medicine 111:15-21, 1989.

28. Velludo MASL, Brunaldi MO, Romanello LMF. Infecções oportunistas do trato digestivo em pacientes com síndrome da imunodeficiência adquirida. Medicina 25:166-179,1992. 